\title{
Malignant tumours of the kidney: imaging strategy
}

\author{
Anne M. Smets • Jan de Kraker
}

Received: 20 January 2010 / Accepted: 30 January 2010

(C) The Author(s) 2010. This article is published with open access at Springerlink.com

\begin{abstract}
Primitive malignant renal tumours comprise 6\% of all childhood cancers. Wilms tumour (WT) or nephroblastoma is the most frequent type accounting for more than $90 \%$. Imaging alone cannot differentiate between these tumours with certainty but it plays an important role in screening, diagnostic workup, assessment of therapy response, preoperative evaluation and follow-up. The outcome of WT after therapy is excellent with an overall survival around $90 \%$. In tumours such as those where the outcome is extremely good, focus can be shifted to a risk-based stratification to maintain excellent outcome in children with low risk tumours while improving quality of life and decreasing toxicity and costs. This review will discuss the imaging issues for WT from the European perspective and briefly discuss the characteristics of other malignant renal tumours occurring in children and new imaging techniques with potential in this matter.
\end{abstract}

Keywords Wilms tumour · Kidney - US · CT · MRI · Renal malignancies $\cdot$ Children $\cdot$ Imaging

\footnotetext{
A. M. Smets $(\bowtie)$

Department of Radiology G1,

Academic Medical Center,

PO Box 22700, Amsterdam 1100 DE, The Netherlands

e-mail: a.m.smets@amc.uva.nl

J. de Kraker

Paediatric Oncology-Academic Medical Center,

Amsterdam, The Netherlands
}

\section{Introduction}

Primitive malignant renal tumours comprise $6 \%$ of all childhood cancers. Wilms tumour (WT) or nephroblastoma is the most frequent type accounting for more than $90 \%$ [1]. All other malignant renal tumours occurring in children such as clear cell sarcoma, rhabdoid tumour and renal cell carcinoma are rare.

Imaging alone cannot differentiate between these tumours with certainty but it plays an important role in screening, diagnostic workup, assessment of therapy response, preoperative evaluation and follow-up. Abdominal US with colour Doppler, chest radiograph, CT and MRI are commonly used for anatomical staging and detection of metastases. New techniques such as $18 \mathrm{~F}$-fluorodeoxyglucose positron emission tomography (FDG-PET) CT and diffusion-weighted imaging (DWI) with MRI are being evaluated for their potential value in the imaging strategy of malignant renal tumours in adults and children.

The outcome of WT after therapy is excellent with an overall survival around $90 \%$ [1]. In tumours such as those where the outcome is extremely good, focus can be shifted to a risk-based stratification to maintain excellent outcome in children with low risk tumours while improving quality of life and decreasing toxicity and costs. At the same time it is important to identify the small group of children with high risk tumours who might benefit from intensified therapy. In this strategy, care should be taken not to increase the burden of invasive or ionizing diagnostic investigations in all children [2].

The therapeutic approach for WT in Europe is different from that in the United States [3, 4]. This review will discuss the imaging issues for WT from the European perspective and briefly discuss the characteristics of other 
malignant renal tumours occurring in children and new imaging techniques with potential in this matter.

\section{Wilms tumour}

\section{Differential diagnosis}

In Europe, WT is treated according to the Société Internationale d'Oncologie Pédiatrique (SIOP) 2001 trial and study protocol [5]. Since biopsy is not required in this protocol, renal tumours with features matching WT are treated accordingly. The diagnosis will be confirmed on pathological examination after preoperative chemotherapy and surgery. The advantages of preoperative chemotherapy have been extensively published in various papers. Analysis of the SIOP-9 study showed that in only $2 \%$ of children preoperative chemotherapy was given inappropriately for benign lesions and in 3\% for other malignant lesions [6]. This seems acceptable since preoperative chemotherapy of short duration (4 weeks) with vincristine and actinomycin-D is not associated with long-term complications and is also effective in most non-Wilms tumours. Vujanic et al. [7] reported the results of pretreatment needle biopsies in children with non-metastatic renal tumours. In $84 \%$ the diagnosis of WT was confirmed and in $12 \%$ it revealed either an alternative renal malignancy or neuroblastoma. In $4 \%$ the specimen was not diagnostic and in $2 \%$ significant complications were described. Whether the pathological information provided by biopsy before therapy is worth the possible complications is not yet proved.

Sometimes a neuroblastoma invading the kidney can mimic a WT. The presence of calcifications and the typical encasement of vessels are signs in favour of neuroblastoma. Elevation of catecholamine levels in $24 \mathrm{~h}$ urine studies will confirm the diagnosis in more than 90\% [8]. Metaiodobenzylguanidine (MIBG)-Iodine ${ }^{123}$ radiotargeting can be very helpful in this situation (Fig. 1).

Imaging at diagnosis

The initial treatment consists of chemotherapy. This will reduce tumour size, hence facilitating subsequent surgery and lowering the risk of intra-operative tumour rupture. Preoperative chemotherapy will also downstage tumours and reduce postoperative therapy. Clinical history, physical examination and diagnostic imaging will assist in differentiating WT from other renal pathology, either benign or malignant. Needle biopsy is recommended in cases of unusual clinical presentation (age above 6 years, urinary infection, septicaemia, psoas inflammation) or unusual imaging findings for WT (calcification, voluminous lymph nodes, renal parenchyma not visible, almost entirely extrarenal mass).

Furthermore, imaging will depict the locoregional extension, metastatic spread and contralateral disease.

Initial imaging should consist of abdominal US with colour Doppler and chest radiograph in 2 projections; frontal

Fig. 1 Axial T2-W fat-supressed MR. 11-month-old boy with a right abdominal mass with a partially intrarenal localization (black arrow) and liver metastases. 123-Iodine-MIBGscan was positive for neuroblastoma

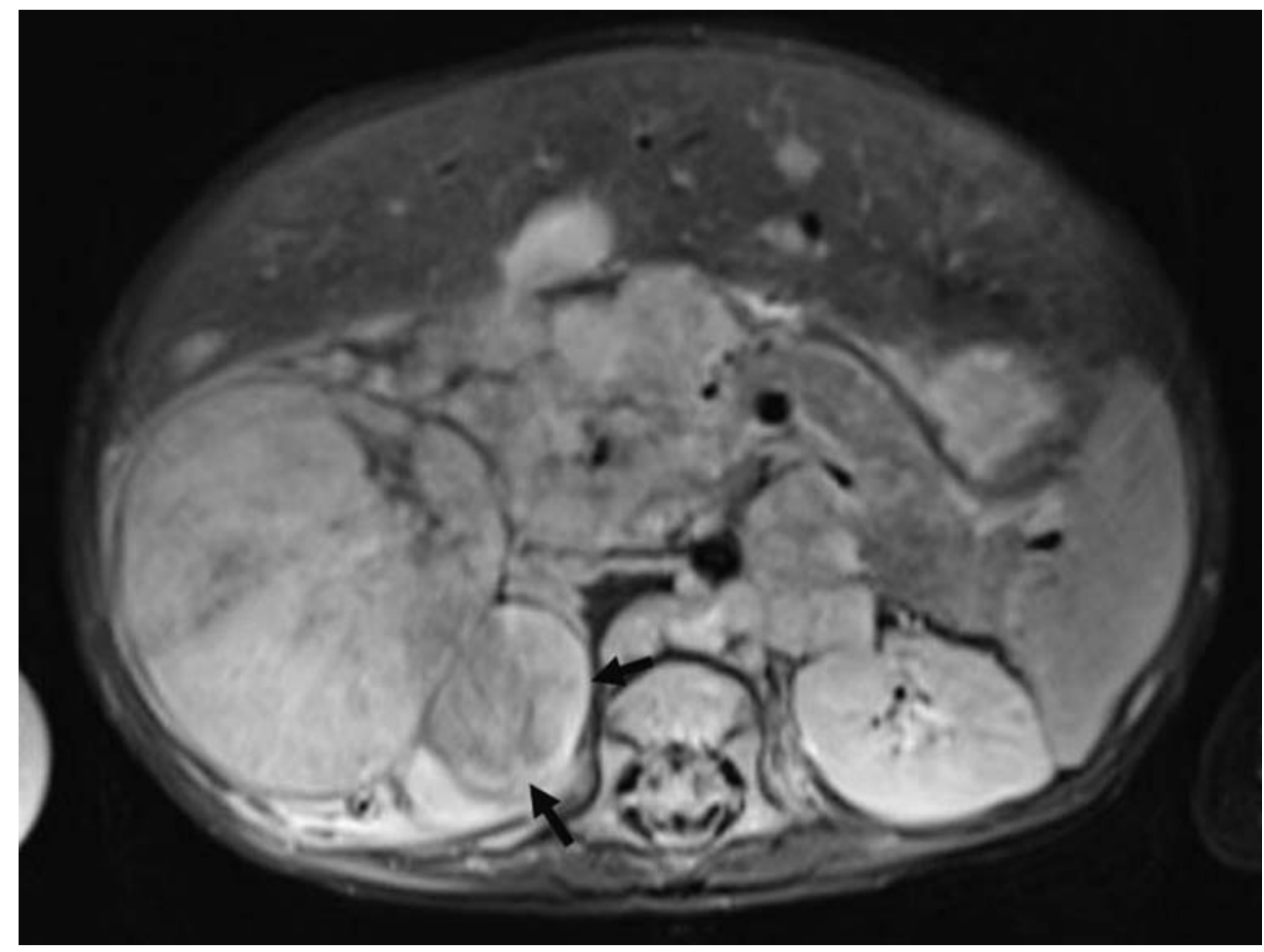


and lateral view. Depending on the size and extension of the tumour and the expertise of the radiologist, complementary abdominal contrast-enhanced CT or MRI will be required to further delineate tumour extension and exclude bilateral disease with certainty (Fig. 2). Although CT is more readily available and faster, MRI is the preferred technique since it does not use ionizing radiation, even though general anaesthesia or sedation is usually necessary in these young children. If there are suspicious pulmonary lesions on chest radiograph, additional chest $\mathrm{CT}$ is recommended.

The primary tumour

WT presents as a large spherical mass, with a mainly intrarenal localization. The tumour can be solid and homogeneous but is usually heterogeneous with areas of haemorrhage, necrosis or cysts. In $<15 \%$ the tumour contains calcifications. The remaining normal kidney tissue can be difficult to detect but is often stretched at the periphery of the tumour (Fig. 3). US is an excellent technique to assess the movement of the mass in relation to its surroundings in order to exclude invasion or adherence to adjacent organs. Measurement of the tumour in three planes is required. If the tumour is large and difficult to delineate from the kidney, kidney and tumour is measured as one. On CT and MRI the tumour enhances less than normal renal tissue making it more conspicuous.

The contralateral kidney

Whenever a renal tumour (presumably a WT) is detected, the contralateral kidney should be examined for synchronous bilateral disease that occurs in $5 \%$ of children. Bilateral disease comprises bilateral WT or bilateral nephrogenic rests

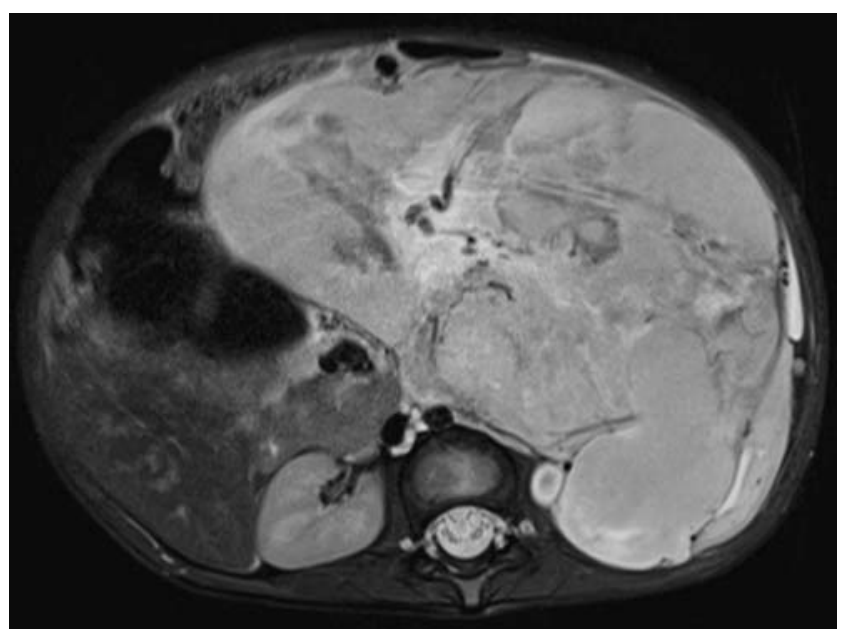

Fig. 2 Axial T2-W fat-suppressed MR shows a large tumour with left renal origin. The mass was too large to be adequately imaged with US alone

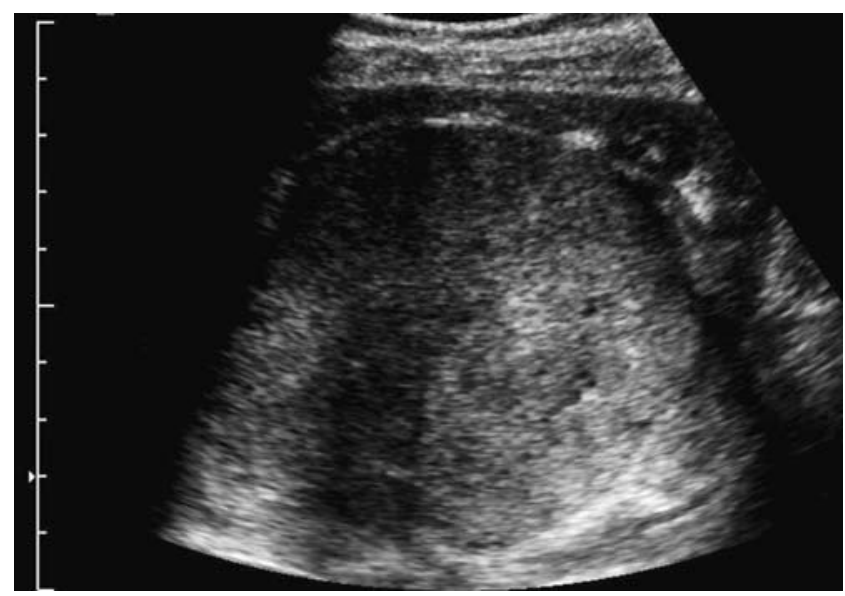

Fig. 3 2.5-year-old girl with a left renal mass. US shows the kidney stretched around the tumour

(NRs) (nephroblastomatosis) and/or WT. Other lesions may mimic bilateral disease, including lymphoma, leukaemia or polycystic kidney disease (Fig. 4). Bilateral disease upstages the patient to stage $\mathrm{V}$ [5]. The aim of treatment then becomes to cure and at the same time preserve as much functional renal tissue as possible by using parenchyma-sparing surgery. Preoperative imaging will then play a crucial role in determining the possibilities of partial nephrectomy.

In case of detection of other non-malignant abnormalities of the other kidney endangering the renal function (e.g. dysplasia, hydronephrosis) or absence of the other kidney, partial nephrectomy must also be considered.

Bilateral disease occurs more frequently in children with NRs in their kidneys. NRs are clusters of embryonal nephroblastic cells that can persist into childhood and have

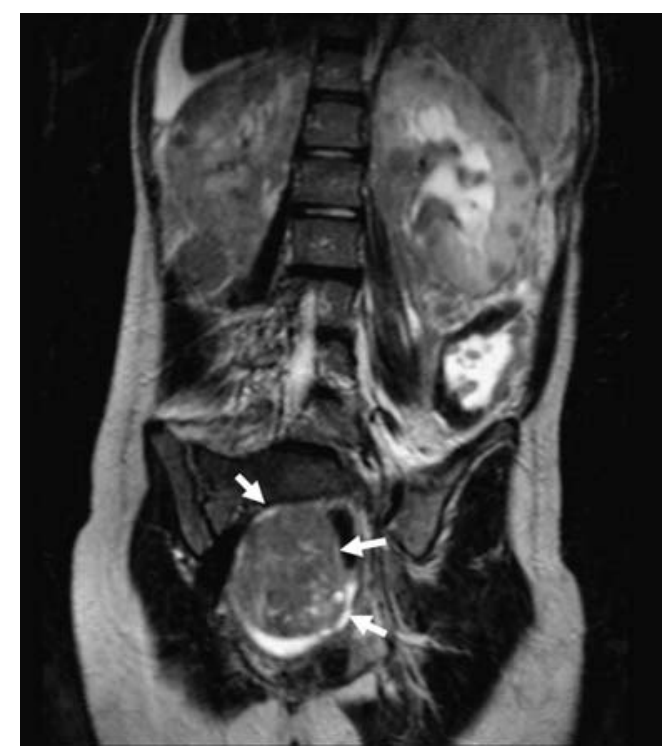

Fig. 4 Twelve-year-old girl with Burkitt lymphoma. Coronal T2-W MR shows in addition to a large abdominal mass (partially shown, white arrows), multiple nodular lesions in both kidneys 
Fig. 5 Two-year-old girl with bilateral nephroblastomatosis. a. Coronal T1-W MR after gadolinium administration shows multiple bilateral round lesions with different patterns of enhancement. b. Axial T2-W MR. The lesions show variable signal intensity. Some of the lesions eventually developed into WT
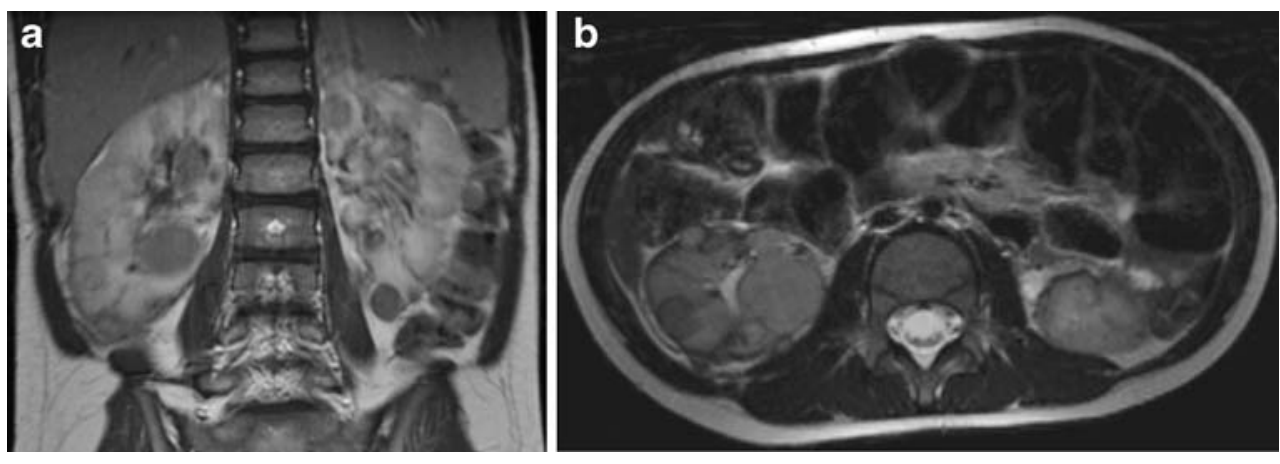

the potential to transform into a malignancy [9]. This makes detection of these often small lesions important. Rohrschneider et al. [10] compared the sensitivity of US, CT and MRI to detect macroscopic NRs and concluded that US is less sensitive than CT and MRI. The value of highresolution US techniques such as tissue harmonic imaging has not yet been evaluated. Nowadays, surgeons no longer explore the contralateral kidney before nephrectomy since imaging is sufficiently capable of detecting small lesions in the contralateral kidney [11, 12]. Differentiating benign NRs from WT is important for it will obviously determine the appropriate management. MRI shows distinctly different characteristics on pre-contrast $\mathrm{T} 2$-weighted images between sclerotic and hyperplastic NRs and WT. This might help in decision making about continuation of chemotherapy or partial nephrectomy [13]. This is particularly useful since pathological examination of a small biopsy specimen cannot discriminate with confidence between benign NRs and WT (Fig. 5).

The management of bilateral disease is still one of the most challenging issues in WT especially when nephron-sparing surgery is not possible due to the location of the lesions.

\section{The rest of the abdomen}

Enlarged lymph nodes are frequently encountered in the region of the tumour. Imaging studies are unable to differentiate between reactive nodes or tumour positive nodes. Hence lymph node biopsies will be performed during surgery, according to the protocol.

WT can metastasise to the liver in $15-20 \%$ of children making meticulous inspection of the hepatic parenchyma necessary.

Although rare, tumour rupture is a major risk factor for abdominal relapse. The reason for preoperative chemotherapy in the SIOP protocol is to decrease the risk of intraoperative tumour rupture, that would upstage the patient to stage III (Table 1). These children will then receive abdominal radiotherapy as part of their postopera-
Table 1 Current SIOP criteria for staging of WT [5]

\section{Stage I}

The tumour is limited to the kidney or surrounded by a fibrous pseudocapsule if outside of the normal contours of the kidney. The renal capsule or pseudocapsule may be infiltrated but the tumour does not reach the outer surface and it is completely resected

The tumour may be protruding into the pelvic system and dipping into the ureter but it is not infiltrating their walls

The vessels of the renal sinus are not involved

Intrarenal vessel involvement may be present

Necrotic tumour or chemotherapy-induced changes in the perirenal fat or the renal sinus do not upstage if completely excised

\section{Stage II}

The tumour extends beyond the kidney or penetrates through the renal capsule and/or fibrous capsule into perirenal fat but is completely resected

Tumour infiltrates the renal sinus and/or invades blood and lymphatic vessels outside the renal parenchyma but is completely resected

Tumour infiltrates adjacent organs or vena cava but is completely resected

\section{Stage III}

a. Incomplete excision of the tumour which extends beyond resection margins (gross or microscopic tumour remains postoperatively)

b. Any abdominal lymph nodes are involved

c. Tumour rupture before or during surgery (irrespective of other criteria for staging)

d. The tumour has penetrated through the peritoneal surface

e. Tumour implants are found on the peritoneal surface

f. Tumour thrombi present at the resection margins of vessels or ureter, transsected or removed piecemeal by the surgeon

g. The tumour has been surgically biopsied (wedge biopsy) prior to preoperative chemotherapy or surgery

The presence of necrotic tumour or chemotherapy-induced changes in a lymph node or at the resection margins is also assigned stage III

\section{Stage IV}

Haematogenous metastases (lung, liver, bone, brain, etc.) or lymph node metastases outside the abdomino-pelvic region

\section{Stage V}

Bilateral renal involvement at diagnosis. Each side should be substaged according to the above classifications 
tive treatment if the tumour shows intermediate or high risk histology. In the SIOP protocol, the local stage is determined by pathological examination of the nephrectomy specimen. However, clinical signs and imaging could also suggest rupture. In the current protocol these findings are not taken into account for the final local staging. Brisse et al. [14] demonstrated from a retrospective study of 57 patients with either clinical and/or CT signs of rupture that detailed analysis of abdominal CT scans may distinguish intraperitoneal from retroperitoneal ruptures. These imaging findings are not always coupled with clinical signs. They suggested that diagnostic features of intraperitoneal rupture should be considered as stage III regardless of other postoperative pathological criteria. On the other hand, they found no increased risk for abdominal relapse in children with isolated retroperitoneal rupture who did not receive abdominal radiotherapy. These findings encourage the possible need to value imaging findings of both retroperitoneal and intraperitoneal rupture when considering postoperative treatment, to prevent respectively over- and under treatment of these children.

Small amounts of non-haemorrhagic peritoneal fluid should not be considered as a sign of rupture: it can correspond to a peritoneal inflammatory reaction to rapid tumour growth or be due to inferior vena cava (IVC) compression or thrombosis [3].

In $4-10 \%$ of children the tumour extends into the IVC [15]. Preoperative chemotherapy will usually at least decrease the size of the tumour thrombus and possibly even induce resolution of the IVC extension. This will reduce the risks of massive haemorrhage or pulmonary embolus during surgery. US in combination with colour Doppler studies is the most reliable modality to examine the patency of the IVC (Fig. 6) [16].
The lungs

Lung metastases are present in $15-20 \%$ of children with WT. In the current SIOP protocol chest radiograph (CXR) (AP or PA and lateral view) is still the basic imaging tool to exclude pulmonary metastases. Complementary chest $\mathrm{CT}$ is recommended only in cases of distinct or suspicious lesions on CXR [5]. In North America, CT has become the routine method of diagnosis for assessment of pulmonary lesions since it is widely accepted that chest $\mathrm{CT}$ is a more sensitive technique for detecting pulmonary nodules than conventional radiographs. However, CT cannot differentiate between small benign pulmonary lesions (that frequently occur in children) and metastases [17-19]. Moreover, it has not been proven that detecting smaller lesions at diagnosis (and possibly also benign lesions) will improve the outcome of WT patients [18, 20-22]. The ongoing discussion around the dilemma between using the most accurate imaging technique to detect pulmonary lesions and avoiding overtreatment in children with a very good overall prognosis has been recently summarized by Owens et al. [3, $23,24]$. In the current SIOP protocol a special clause is made for patients with lung lesions detected on chest CT and not on CXR. These lesions are called CT-only lung metastases. The SIOP protocol proscribes that these lesions should be ignored and the patients with CT-only nodules should be treated as if without lung metastases (double agent instead of three-drug chemotherapy and no lung irradiation). One of the aims of the study is to evaluate the outcome of these patients with CT-only metastases. If it is not different from patients without CT-only lesions, with same stage and histology, it could make detection of these lesions unnecessary and hence chest CT redundant at diagnosis. It will also prevent these patients from receiving

Fig. 6 Five-year-old girl with left sided WT. Abdominal US shows a large tumour thrombus in the IVC (white arrows)

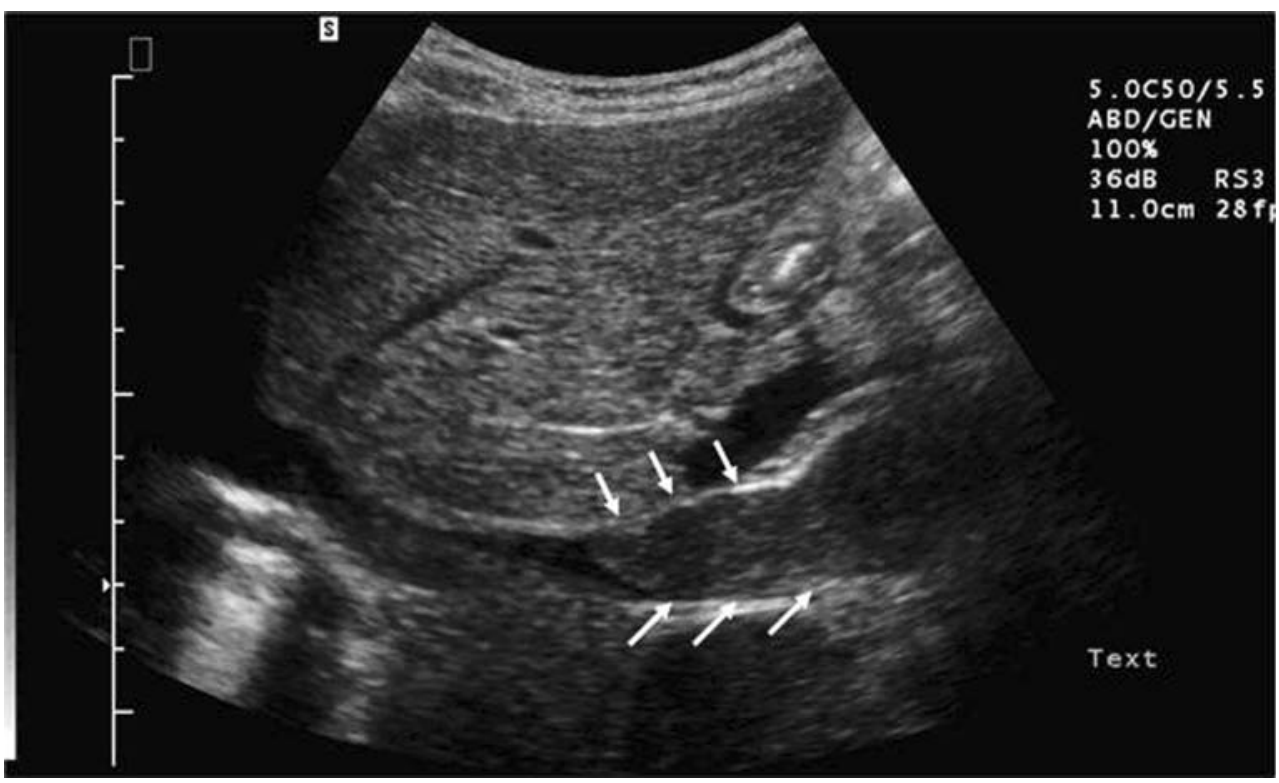


more toxic treatment with more long-term side effects. If on the other hand, patients with CT-only metastases do worse, chest CT should undoubtedly become the mandatory diagnostic tool in the imaging strategy of WT.

\section{Preoperative imaging}

Most tumours decrease in size during preoperative chemotherapy and/or show changes in appearance indicating necrosis. The tumour rarely increases in size (5\%), predicting poorer event-free and overall survival [25]. The surgeon needs to know the extent of the tumour, its location within the kidney and its relation to the central vessels, diaphragm, liver, spleen, pancreas and adrenal glands before operation. Enlarged lymph nodes, intra-abdominal metastases (mainly liver) and thrombus in renal vein, IVC or right atrium must be looked for. US is often sufficient to answer these questions and in difficult cases it is recommended to perform the examination in the presence of the surgeon. If US does not provide satisfactory information, MRI is the preferred complementary modality because of the lack of ionizing radiation.

Kidney-sparing surgery is indicated in cases of bilateral disease, underlying urologic or nephrologic disease (e.g. horseshoe kidney, single kidney) or genetic syndromes with an increased risk of WT. In this context multiplanar acquisitions with MRI are extremely helpful in depicting the surgical possibilities and margins. Partial nephrectomy can also be considered in cases of simple unilateral disease; the SIOP protocol mentions contraindications for this procedure [26]. Some of these can be detected by the radiologist: tumour infiltrating into extrarenal structures, intra-abdominal metastases or enlarged lymph nodes, thrombus in the renal vein or IVC, tumour involving more than $1 / 3$ of the kidney, multifocal tumour, central location and involvement of calyces.

Intraoperative US may also assist in defining the tumour margins more accurately.

\section{Follow-up}

Tumour relapse in WT is infrequent and typically occurs in the first 2 years after therapy. Considering the overall good prognosis of WT, imaging after treatment should also be minimally invasive and minimally harmful. Since recurrence of WT can be cured, it is important to detect it (Fig. 7). Children are followed with CXR and abdominal US. Abdominal US is recommended at a higher frequency in cases of metastatic disease in complete remission, if nephrogenic rests are present, in cases of bilateral disease and after partial nephrectomy, than for children with nonmetastatic disease at diagnosis. There is no evidence or consensus about the frequency of follow-up studies.

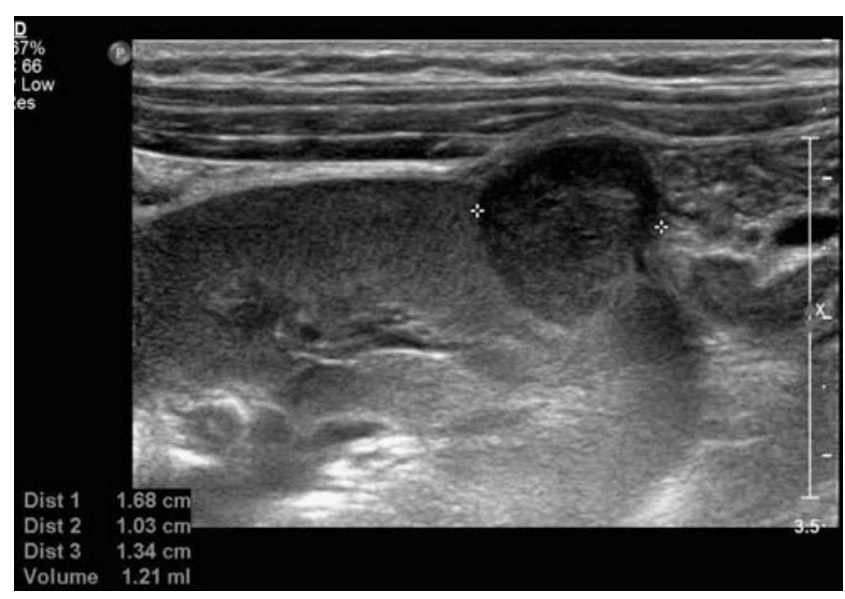

Fig. 7 Nine-year-old girl with recurrence detected on routine US. She had been in complete remission over a 7 year period after a stage 2 intermediate risk WT. Courtesy of Dr. S. Robben

\section{Screening}

Most WT occur in otherwise healthy children. A small number occur in children with genetic syndromes. Scott et al. [27] have produced guidelines for WT surveillance based on a review of current practice and available evidence. In summary, they recommend using renal US every 3-4 months until 5 or 7 years of age to screen children with a risk of developing WT $>5 \%$, depending on the syndrome. These children should first be offered a review by a clinical geneticist and detected lesions should be managed at a specialist centre.

\section{Other renal malignancies of childhood}

\section{Clear cell sarcoma}

Clear cell sarcoma of the kidney (CCSK) occurs in children with a peak age of incidence similar to that of WT. They account for approximately $4 \%$ of childhood renal tumours. Metastatic spread is wider than in WT; to bone, brain, liver, lymph nodes and lungs, in some cases long after nephrectomy. Radioisotope bone scan (99 m-Technetium-methylene diphosphate), MRI of the brain and chest $\mathrm{CT}$ are recommended for staging. Children with CCSK are at risk for relapse for a longer period of time than WT and they have a poorer outcome with long-term survival rates of 60 $70 \%$ [1]. Imaging studies cannot distinguish between CCSK and WT (Fig. 8) [1, 28].

Rhabdoid tumours of the kidney

Rhabdoid tumours of the kidney (RTK) account for $1-2 \%$ of renal malignancies in childhood. RTK is a highly 


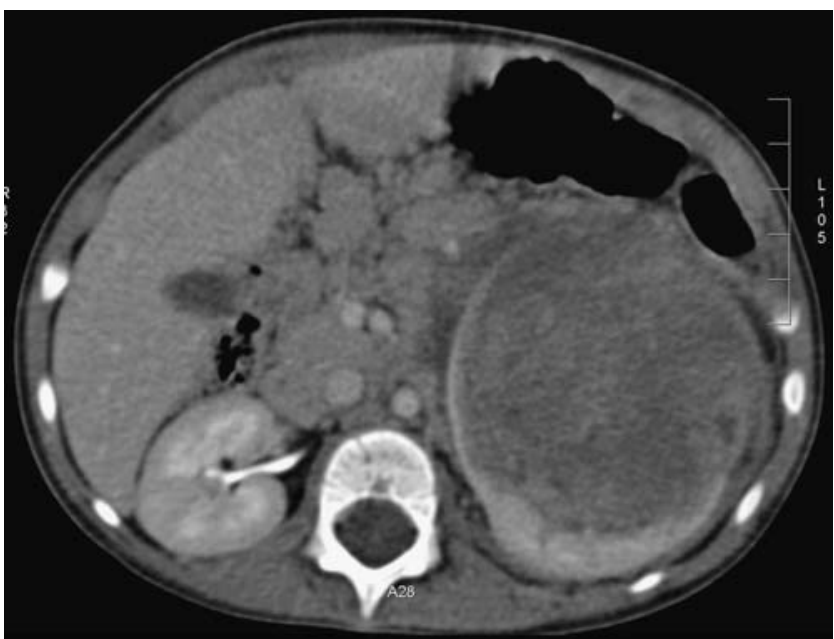

Fig. 8 Two-year-old girl with a clear cell sarcoma in the left kidney. Contrast-enhanced abdominal CT shows the mass indistinguishable from a WT on imaging studies

aggressive entity occurring exclusively in children and mainly in infants. Brain metastases or synchronous primary intracranial masses are often present at diagnosis. The brain lesion is usually near the midline and often in the posterior fossa. Survival is often $<1$ year from diagnosis. Imaging studies demonstrate a large, centrally located, heterogeneous soft-tissue mass involving the renal hilum. The appearance may closely resemble WT but subcapsular fluid collections, tumour lobules separated by areas of necrosis or haemorrhage and linear calcifications outlining tumour lobules can suggest the diagnosis of RTK. Renal vein invasion is common. RTK can metastasise to lungs, liver and brain $[28,29]$.

\section{Renal cell carcinoma}

Renal cell carcinoma (RCC) is the most common renal tumour in adults. It presents very rarely in childhood (1\%). Children with tuberous sclerosis or von Hippel-Lindau disease or prior chemotherapy for cancer are at risk [30]. There also seems to be a higher incidence in children who have been previously treated for neuroblastoma [31]. Compared to WT, RCC more often presents bilaterally and is more likely to metastasize to bone. Calcifications are more frequently seen than in WT [28].

In contrast to adults, locoregional nodal spread seems not to worsen the prognosis [32].

\section{Renal medullary carcinoma}

Renal medullary carcinoma is a highly malignant tumour of epithelial origin occurring almost exclusively in adolescents and young adults with sickle cell trait or sickle cell disease, but not homozygous sickle cell disease [33, 34]. The age range at presentation is 10-39 years (mean age 20). Imaging shows a centrally located infiltrative lesion invading the renal sinus. The tumour is heterogeneous and hypovascular. Intratumoural and subcapsular haemorrhage are common. Calcifications have not been described. Renal vein thrombosis or encasement is a common feature [35]. The tumour metastasises to lungs and liver and responds poorly to chemotherapy or radiation therapy with a mean survival of 15 weeks from diagnosis.

\section{Congenital mesoblastic nephroma}

Congenital mesoblastic nephroma $(\mathrm{CMN})$ is the most common solid tumour in the newborn. Below the age of 3 months, $72 \%$ of renal tumours are CMN. At 6 months this percentage decreases to $16 \%$ [36]. Although it used to be considered a congenital WT, it is now known to be a distinct entity. It is usually detected within the first 3 months or at least first year of life. Some cases are identified prenatally. Imaging studies demonstrate a large solid intrarenal mass that typically involves the renal sinus. It may contain cystic, haemorrhagic and necrotic components. Local infiltration of the perirenal tissues is common. Nephrectomy alone with a wide surgical margin is usually sufficient for cure. Local recurrence and spread to lungs, brain or bones is rare [28].

\section{Primitive neuroectodermal tumour}

Primitive neuroectodermal tumour (PNET) of the kidney is an extremely rare malignant tumour often presenting in an advanced stage. Renal PNET behaves more aggressively than PNET at other sites. It has poor response to chemotherapy. Imaging studies show an infiltrative mass that may present with calcifications and areas of necrosis. The tumour can extend in the renal vein or IVC $[37,38]$.

\section{New imaging techniques}

\section{FDG-PET}

FDG-PET has been established for a number of diseases in adults. In combination with $\mathrm{CT}$ the increased metabolic activity can be anatomically localized and hence provides more accurate information for staging; differentiating benign from malignant disease and monitoring response to treatment.

The applications in children are being further explored and are promising in many areas. Although PET-scanning is noninvasive, it does involve exposure to ionizing radiation and the total dose is significant, usually around $10 \mathrm{mSv}$. Uptake of FDG by WT has been described but a definitive 
role has not been established. For differentiating lung metastases from benign pulmonary lesions PET-CT fails since both conditions may involve increased glucose metabolism. Furthermore, the current resolution of PETCT does not allow detection of micrometastases [39].

In a study by Misch et al. [40] 12 children with WT were examined with FDG-PET. Their results suggest that the usefulness of this technique could lie not in initial staging but in post-therapeutic imaging, non-invasive tumour characterisation and restaging in cases of recurrent disease.

Normal excretion of FDG through the kidney can be a limiting factor in detection of small renal tumours.

\section{Quantitative MRI}

DWI has been widely accepted as a powerful imaging technique in neuroradiology. With advances in MRI technology, diffusion-weighted sequences can now also be used in body imaging with a minimal increase in imaging time. For renal tumours in adults it is not yet possible to confidently distinguish between benign and malignant renal disease on the basis of quantitative assessment of ADC measurements alone because of considerable overlap between ADC values of benign and malignant disease [41, 42]. However, DWI can help differentiate between viable and necrotic areas within tumours that can be very useful in selecting optimal biopsy sites and detecting viable tumour after treatment [41]. Abdominal DWI is feasible in children as well. Humphries et al. [43] examined three children with nephroblastoma with DW techniques on clinical 1.5-T MR units. They confirm that DWI in children can help in guiding biopsy away from necrotic areas and suggesting poor response to chemotherapy before tumour shrinkage is expected. However, DWI was unable to differentiate between benign and malignant lesions based on ADC values alone [43].

\section{Conclusion}

For WT, a renal malignancy with an excellent prognosis, the imaging guidelines for the European therapeutic approach are outlined in the SIOP 2001 protocol. Soon this trial will be closed and the results will be evaluated. In the meantime a multidisciplinary team of physicians involved in the management of children with WT is encouraged to reflect on strategies for a new protocol in order to answer remaining questions and assist in a new risk-based approach. There is unquestionably a distinct task for radiologists who are involved in national and international paediatric oncology study groups. They will have to face several challenges including selecting the most accurate techniques (preferably non-ionizing and noninva- sive) and choosing the right timing to perform them so that the minority of children who might benefit from different or more intensive therapy can be established reliably and cost-effectively.

Hopefully, the results of the SIOP 2001 trial will bring some clarity about the value of chest $\mathrm{CT}$ in the detection of pulmonary metastases. The future will tell if quantitative MRI techniques and/or FDG-PET will appear helpful in discriminating renal lesions and facilitate the management of bilateral disease.

For other less common childhood malignant renal tumours such as clear cell sarcoma, for which a structured diagnostic work-up is lacking, there is a need for evidencebased strategies for staging and follow-up.

Acknowledgements The author thanks Eline Deurloo and Koen Smets for helping with manuscript preparation.

Open Access This article is distributed under the terms of the Creative Commons Attribution Noncommercial License which permits any noncommercial use, distribution, and reproduction in any medium, provided the original author(s) and source are credited.

\section{References}

1. Pastore G, Znaor A, Spreafico F et al (2006) Malignant renal tumours incidence and survival in European children (19781997): report from the Automated Childhood Cancer Information System project. Eur J Cancer 42:2103-2114

2. Voss SD, Reaman GH, Kaste SC et al (2009) The ALARA concept in pediatric oncology. Pediatr Radiol 39:1142-1146

3. Brisse HJ, Smets AM, Kaste SC et al (2008) Imaging in unilateral Wilms tumour. Pediatr Radiol 38:18-29

4. Kaste SC, Dome JS, Babyn PS et al (2008) Wilms tumour: prognostic factors, staging, therapy and late effects. Pediatr Radiol 38:2-17

5. SIOP (2001) Nephroblastoma clinical trial and study protocol. In: Oncology ISoP (ed), pp 1-170

6. Tournade MF, Com-Nougue C, de Kraker J et al (2001) Optimal duration of preoperative therapy in unilateral and nonmetastatic Wilms' tumor in children older than 6 months: results of the Ninth International Society of Pediatric Oncology Wilms' Tumor Trial and Study. J Clin Oncol 19:488-500

7. Vujanic GM, Kelsey A, Mitchell C et al (2003) The role of biopsy in the diagnosis of renal tumors of childhood: results of the UKCCSG Wilms tumor study 3. Med Pediatr Oncol 40:18-22

8. Strenger V, Kerbl R, Dornbusch HJ et al (2007) Diagnostic and prognostic impact of urinary catecholamines in neuroblastoma patients. Pediatr Blood Cancer 48:504-509

9. Owens CM, Brisse HJ, Olsen OE et al (2008) Bilateral disease and new trends in Wilms tumour. Pediatr Radiol 38:30-39

10. Rohrschneider WK, Weirich A, Rieden K et al (1998) US, CT and MR imaging characteristics of nephroblastomatosis. Pediatr Radiol 28:435-443

11. Ritchey ML, Shamberger RC, Hamilton T et al (2005) Fate of bilateral renal lesions missed on preoperative imaging: a report from the National Wilms Tumor Study Group. J Urol 174:1519-1521

12. Hamilton TE, Ritchey ML, Argani P et al (2008) Synchronous bilateral Wilm's tumor with complete radiographic response managed without surgical resection: a report from the National Wilm's Tumor Study 4. J Pediatr Surg 43:1982-1984 
13. Gylys-Morin V, Hoffer FA, Kozakewich H et al (1993) Wilms tumor and nephroblastomatosis: imaging characteristics at gadolinium-enhanced MR imaging. Radiology 188:517-521

14. Brisse HJ, Schleiermacher G, Sarnacki S et al (2008) Preoperative Wilms tumor rupture: a retrospective study of 57 patients. Cancer 113:202-213

15. Lall A, Pritchard-Jones K, Walker J et al (2006) Wilms' tumor with intracaval thrombus in the UK Children's Cancer Study Group UKW3 trial. J Pediatr Surg 41:382-387

16. Ritchey ML, Kelalis PP, Breslow N et al (1988) Intracaval and atrial involvement with nephroblastoma: review of National Wilms Tumor Study-3. J Urol 140:1113-1118

17. Cohen M, Smith WL, Weetman R et al (1981) Pulmonary pseudometastases in children with malignant tumors. Radiology 141:371-374

18. Wilimas JA, Douglass EC, Magill HL et al (1988) Significance of pulmonary computed tomography at diagnosis in Wilms' tumor. $\mathrm{J}$ Clin Oncol 6:1144-1146

19. Grundy P, Perlman E, Rosen NS et al (2005) Current issues in Wilms tumor management. Curr Probl Cancer 29:221-260

20. Owens CM, Veys PA, Pritchard J et al (2002) Role of chest computed tomography at diagnosis in the management of Wilms' tumor: a study by the United Kingdom Children's Cancer Study Group. J Clin Oncol 20:2768-2773

21. Meisel JA, Guthrie KA, Breslow NE et al (1999) Significance and management of computed tomography detected pulmonary nodules: a report from the National Wilms Tumor Study Group. Int J Radiat Oncol Biol Phys 44:579-585

22. Wootton-Gorges SL, Albano EA, Riggs JM et al (2000) Chest radiography versus chest $\mathrm{CT}$ in the evaluation for pulmonary metastases in patients with Wilms' tumor: a retrospective review. Pediatr Radiol 30:533-537

23. Cohen M (2008) Wilms tumor: a new method for quantification of lung metastatic tumor burden. Pediatr Radiol 38:817-818

24. Owens CM, Kaste SC, Smets A et al (2008) Reply. Pediatr Radiol 38:819-820

25. Ora I, van Tinteren H, Bergeron C et al (2007) Progression of localised Wilms' tumour during preoperative chemotherapy is an independent prognostic factor: a report from the SIOP 93-01 nephroblastoma trial and study. Eur J Cancer 43:131-136

26. Zani A, Schiavetti A, Gambino M et al (2005) Long-term outcome of nephron sparing surgery and simple nephrectomy for unilateral localized Wilms tumor. J Urol 173:946-948

27. Scott RH, Walker L, Olsen OE et al (2006) Surveillance for Wilms tumour in at-risk children: pragmatic recommendations for best practice. Arch Dis Child 91:995-999
28. Lowe LH, Isuani BH, Heller RM et al (2000) Pediatric renal masses: Wilms tumor and beyond. Radiographics 20:1585-1603

29. McHugh K (2007) Renal and adrenal tumours in children. Cancer Imaging 7:41-51

30. Sausville JE, Hernandez DJ, Argani P et al (2009) Pediatric renal cell carcinoma. J Pediatr Urol 5:308-314

31. Bassal M, Mertens AC, Taylor L et al (2006) Risk of selected subsequent carcinomas in survivors of childhood cancer: a report from the Childhood Cancer Survivor Study. J Clin Oncol 24:476-483

32. Geller JI, Dome JS (2004) Local lymph node involvement does not predict poor outcome in pediatric renal cell carcinoma. Cancer 101:1575-1583

33. Swartz MA, Karth J, Schneider DT et al (2002) Renal medullary carcinoma: clinical, pathologic, immunohistochemical, and genetic analysis with pathogenetic implications. Urology 60:1083-1089

34. Davidson AJ, Choyke PL, Hartman DS et al (1995) Renal medullary carcinoma associated with sickle cell trait: radiologic findings. Radiology 195:83-85

35. Blitman NM, Berkenblit RG, Rozenblit AM et al (2005) Renal medullary carcinoma: CT and MRI features. AJR 185:268-272

36. van den Heuvel-Eibrink MM, Grundy P, Graf N et al (2008) Characteristics and survival of 750 children diagnosed with a renal tumor in the first seven months of life: a collaborative study by the SIOP/GPOH/SFOP, NWTSG, and UKCCSG Wilms tumor study groups. Pediatr Blood Cancer 50:1130-1134

37. Siegel MJ, Chung EM (2008) Wilms' tumor and other pediatric renal masses. Magn Reson Imaging Clin N Am 16:479497, vi

38. Citak EC, Oguz A, Karadeniz C et al (2009) Primitive neuroectodermal tumor of the kidney in a child. Pediatr Hematol Oncol $26: 481-486$

39. Kaste SC (2008) 18F-PET-CT in extracranial paediatric oncology: when and for whom is it useful? Pediatr Radiol 38:S459-S466

40. Misch D, Steffen IG, Schonberger S et al (2008) Use of positron emission tomography for staging, preoperative response assessment and posttherapeutic evaluation in children with Wilms tumour. Eur J Nucl Med Mol Imaging 35:1642-1650

41. Saremi F, Knoll AN, Bendavid OJ et al (2009) Characterization of genitourinary lesions with diffusion-weighted imaging. Radiographics 29:1295-1317

42. Zhang J, Tehrani YM, Wang L et al (2008) Renal masses: characterization with diffusion-weighted MR imaging-a preliminary experience. Radiology 247:458-464

43. Humphries PD, Sebire NJ, Siegel MJ et al (2007) Tumors in pediatric patients at diffusion-weighted MR imaging: apparent diffusion coefficient and tumor cellularity. Radiology 245:848-854 